\title{
Zero minors of the neutrino mass matrix
}

\author{
E. I. Lashin ${ }^{1,2,3 *}$ and N. Chamoun ${ }^{1,4 \dagger}$, \\ 1 The Abdus Salam ICTP, P.O. Box 586, 34100 Trieste, Italy. \\ 2 Ain Shams University, Faculty of Science, Cairo 11566, Egypt. \\ ${ }^{3}$ Department of physics and Astronomy, College of Science, King Saud University, Riyadh, Saudi Arabia, \\ ${ }^{4}$ Physics Department, HIAST, P.O.Box 31983, Damascus, Syria.
}

October 29, 2018

\begin{abstract}
We examine the possibility that a certain class of neutrino mass matrices, namely those with two independent vanishing minors in the flavor basis, regardless of being invertible or not, is sufficient to describe current data. We compute generic formulae for the ratios of the neutrino masses and for the Majorana phases. We find that seven textures with two vanishing minors can accommodate the experimental data. We present an estimate of the mass matrix for these patterns. All the possible textures can be dynamically generated through the seesaw mechanism augmented with a discrete Abelian symmetry.
\end{abstract}

PACS numbers: 14.60.Pq; 11.30.Hv; 14.60.St

\section{Introduction}

The results of the Super-Kamiokande [1] on the solar neutrino $\left(\nu_{e}\right)$ deficit and the atmospheric neutrino $\left(\nu_{\mu}\right)$ anomaly can be interpreted if attributed to neutrino oscillations, which in turn can naturally occur if neutrinos are massive and lepton flavors are mixed [2]. Massive neutrinos are commonly believed to be Majorana particles, and at low energy scales, the phenomenology of lepton masses and flavor mixing can be formulated in terms of the charged lepton mass matrix $M_{l}$ and the (effective) neutrino mass matrix $M_{\nu}$. They totally involve twelve physical parameters: three masses of charged leptons $\left(m_{e}, m_{\mu}\right.$ and $m_{\tau}$ ), which have precisely been measured [3]; three masses of neutrinos $\left(m_{1}, m_{2}\right.$ and $\left.m_{3}\right)$, whose relative sizes have been estimated from solar and atmospheric neutrino oscillations $[4,5,6]$; three angles of flavor mixing $\left(\theta_{x}, \theta_{y}\right.$ and $\left.\theta_{z}\right)$, whose values have been constrained to an acceptable accuracy also from solar, atmospheric and reactor neutrino oscillations [4, 5, 6, 7]; and three phases of $\mathrm{CP}$ violation (two Majorana-type $\rho, \sigma$ and one Dirac-type $\delta$ ), which are completely unrestricted by current neutrino data.

One can work in the flavor basis which identifies the flavor eigenstates of the charged leptons with their mass eigenstates, so that $M_{\nu}$ will contain nine free parameters. However, in order to account for the experimental constraints, we need extra assumptions for $M_{\nu}$. The general idea is to assume that some independent matrix elements of $M_{\nu}$ are actually dependent upon one another, caused by an underlying (broken) flavor symmetry. In particular, this dependence becomes very simple and transparent, if the relevant matrix elements are exactly equal to zero.

\footnotetext{
*elashin@ictp.it

†nchamoun@hiast.edu.sy
} 
In fact, general categories of zero-textures were studied: Out of the twenty possible patterns of $M_{\nu}$ with three independent vanishing entries, none is allowed by current neutrino oscillation data, while of the fifteen possible patterns of $M_{\nu}$ with two independent vanishing entries, there are nine patterns which are found to be compatible with current experimental data (albeit two of them are only marginally allowed $[8,9])$. A detailed phenomenological study for the two-textures zero was given in [10]. As to the six possible one-zero textures of $M_{\nu}$, a phenomenological analysis in the general case was given in [11], whereas a specific model having two heavy right handed neutrinos in was suggested in [12] led to a vanishing mass eigenvalue.

In this paper, we adopt a new texture of two independent vanishing minors in the mass matrix $M_{\nu}$. Since a zero-element can be viewed as a zero-determinant of a $1 \times 1$ sub-matrix, then the pattern with vanishing minors (which are determinants of $2 \times 2$ sub-matrices) can be viewed as a generalization of the zero-textures belonging to the same category of matrices with vanishing sub-determinants. In fact, a zero minor in $M_{\nu}$ can be related to a zero entry in the Majorana mass matrix of the right handed singlet neutrinos $M_{R}$ in the canonical see-saw mechanism:

$$
M_{\nu}=M_{D} M_{R}^{-1} M_{D}^{T}
$$

where $M_{D}$ is the Dirac neutrino mass matrix. It was argued [13] that the zeros of $M_{R}$ have a deeper theoretical meaning than the texture zero of $M_{\nu}$ and that if $M_{D}$ is diagonal then texture zeros of $M_{R}$ are reflected in $M_{\nu}$ as zero minors. To keep $M_{D}$ diagonal and to maintain the form of the studied pattern of $M_{\nu}$, a suitable family symmetry $\left(A_{4}\right)$ was introduced in [14].

The work of [15] investigated viable textures with two zeros in the inverted neutronino mass matrix $M_{\nu}^{-1}$ and, using abelian symmetries with one or two heavy scalar singlets, realizations of these textures were constructed. Our work assuming two vanishing minors is somehow different from the work of [15] in the following three points:

- It is true that a vanishing cofactor of the neutrino mass matrix implies a zero in the inverse neutrino mass matrix, but the equivalence comes when the inverse exists. Differently from [15], we did not assume that the neutrino mass matrix is invertible, and in some cases in our work we could make one of the masses $m_{1}$ or $m_{3}$ exactly equal to zero.

- The motivation for studying the texture of vanishing minors is somehow different form that for studying the zero texture in the inverse mass matrix, in the sense that we are generalizing the zero texture in a non trivial way, regardless of its relation to zero textures in other matrices. In [16], textures containing two independent traceless $2 \times 2$ sub-matrices were studied, while here we study textures of two independent 'determinant-less' $2 \times 2$ sub-matrices.

- The phenomenological analysis in this paper contains different details from that of [15]. The strategy we followed consisted of the fact that putting two minors equal to zero gives us four real conditions, so with plausible values for five given input data parameters (the three mixing angles $\theta_{x}, \theta_{y}$ and $\theta_{z}$, and the Dirac CP-violating phase $\delta$, and one input taken to be the solar neutrino mass-squared difference $\Delta m_{\text {sol }}^{2}$ ), one should be able to test the validity of the model to fit the other data. We have varied the known neutrino parameters in their allowed ranges and checked whether vanishing minors are allowed. Whereas in [16], eight textures out of the possible fifteen textures of vanishing two-subtraces were shown to be allowed by experimental data, here we find that seven zero-minor textures, divided into three categories, are able to accommodate the current data, with three patterns showing only normal type hierarchy. One, and only one, acceptable two-vanishing minor texture of singular neutrino mass matrix can be obtained, when $m_{3}=0$ and $\theta_{z}=0$, in three patterns which, otherwise, are failing. 
In accordance with [15], we give an order of magnitude to the neutrino mass matrix, for acceptable values of the input parameters, and find that four acceptable patterns of the two-zeroes textures can be reproduced, with analysis conforming to [9].

The plan of the paper is as follows: in section 2, we review the standard notation for the three-flavor neutrino oscillations and its relation to the experimental constraints. In section 3 , we present the texture of $M_{\nu}$ with two independent vanishing minors and compute the expressions of the two neutrino mass ratios and the Majaorana phases. We classify the patterns and present the results and the phenomenological analysis of each case in sections $4-8$. The symmetry realization of all models is presented in section 9 . We end up by conclusions in section 10 .

\section{Standard notation}

In the flavor basis where the charged lepton mass matrix is diagonal, the symmetric neutrino mass matrix $M_{\nu}$ can be diagonalized by a unitary transformation,

$$
V^{\dagger} M_{\nu} V^{*}=\left(\begin{array}{ccc}
m_{1} & 0 & 0 \\
0 & m_{2} & 0 \\
0 & 0 & m_{3}
\end{array}\right)
$$

with $m_{i}$ (for $i=1,2,3$ ) real and positive, while the lepton flavor mixing matrix $V$ can be written as a product of a Dirac-type flavor mixing matrix $U$ (consisting of three mixing angles and one CP-violating phase) and a diagonal phase matrix $P$ (consisting of two nontrivial Majorana phases): $V=U P$ where $P=\operatorname{diag}\left(e^{i \rho}, e^{i \sigma}, 1\right)$. With

$$
\lambda_{1}=m_{1} e^{2 i \rho}, \quad \lambda_{2}=m_{2} e^{2 i \sigma}, \quad \lambda_{3}=m_{3},
$$

we may rewrite $M_{\nu}$ as

$$
M_{\nu}=U\left(\begin{array}{ccc}
\lambda_{1} & 0 & 0 \\
0 & \lambda_{2} & 0 \\
0 & 0 & \lambda_{3}
\end{array}\right) U^{T}
$$

As to the matrix $U$, and taking the indices $(1,2,3)$ to refer to the flavors $(e, \mu, \tau)$ respectively, it can be parameterized as [9]:

$$
U=\left(\begin{array}{ccc}
c_{x} c_{z} & s_{x} c_{z} & s_{z} \\
-c_{x} s_{y} s_{z}-s_{x} c_{y} e^{-i \delta} & -s_{x} s_{y} s_{z}+c_{x} c_{y} e^{-i \delta} & s_{y} c_{z} \\
-c_{x} c_{y} s_{z}+s_{x} s_{y} e^{-i \delta} & -s_{x} c_{y} s_{z}-c_{x} s_{y} e^{-i \delta} & c_{y} c_{z}
\end{array}\right),
$$

where $s_{x} \equiv \sin \theta_{x}, c_{x} \equiv \cos \theta_{x}$ (we will use later also the notation $t_{x}$ for $\tan x$ ), and so on. From eq.(4), the mass matrix elements take the forms:

$$
\begin{aligned}
M_{\nu 11}= & m_{1} c_{x}^{2} c_{z}^{2} e^{2 i \rho}+m_{2} s_{x}^{2} c_{z}^{2} e^{2 i \sigma}+m_{3} s_{z}^{2}, \\
M_{\nu 12}= & m_{1}\left(-c_{z} s_{z} c_{x}^{2} s_{y} e^{2 i \rho}-c_{z} c_{x} s_{x} c_{y} e^{i(2 \rho-\delta)}\right)+m_{2}\left(-c_{z} s_{z} s_{x}^{2} s_{y} e^{2 i \sigma}+c_{z} c_{x} s_{x} c_{y} e^{i(2 \sigma-\delta)}\right)+m_{3} c_{z} s_{z} s_{y}, \\
M_{\nu 13}= & m_{1}\left(-c_{z} s_{z} c_{x}^{2} c_{y} e^{2 i \rho}+c_{z} c_{x} s_{x} s_{y} e^{i(2 \rho-\delta)}\right)+m_{2}\left(-c_{z} s_{z} s_{x}^{2} c_{y} e^{2 i \sigma}-c_{z} c_{x} s_{x} s_{y} e^{i(2 \sigma-\delta)}\right)+m_{3} c_{z} s_{z} c_{y}, \\
M_{\nu 22}= & m_{1}\left(c_{x} s_{z} s_{y} e^{i \rho}+c_{y} s_{x} e^{i(\rho-\delta)}\right)^{2}+m_{2}\left(s_{x} s_{z} s_{y} e^{i \sigma}-c_{y} c_{x} e^{i(\sigma-\delta)}\right)^{2}+m_{3} c_{z}^{2} s_{y}^{2}, \\
M_{\nu 23}= & m_{1}\left(c_{x}^{2} c_{y} s_{y} s_{z}^{2} e^{2 i \rho}+s_{z} c_{x} s_{x}\left(c_{y}^{2}-s_{y}^{2}\right) e^{i(2 \rho-\delta)}-c_{y} s_{y} s_{x}^{2} e^{2 i(\rho-\delta)}\right) \\
& +m_{2}\left(s_{x}^{2} c_{y} s_{y} s_{z}^{2} e^{2 i \sigma}+s_{z} c_{x} s_{x}\left(s_{y}^{2}-c_{y}^{2}\right) e^{i(2 \sigma-\delta)}-c_{y} s_{y} c_{x}^{2} e^{2 i(\sigma-\delta)}\right)+m_{3} s_{y} c_{y} c_{z}^{2}, \\
M_{\nu 33}= & m_{1}\left(c_{x} s_{z} c_{y} e^{i \rho}-s_{y} s_{x} e^{i(\rho-\delta)}\right)^{2}+m_{2}\left(s_{x} s_{z} c_{y} e^{i \sigma}+s_{y} c_{x} e^{i(\sigma-\delta)}\right)^{2}+m_{3} c_{z}^{2} c_{y}^{2} .
\end{aligned}
$$


If we denote by $\epsilon$ to the transposition on the set 1,2,3 which swaps the two indices 2 and 3 , then under the symmetry $T$ on the parameters defined by :

$$
T \quad: \quad \theta_{y} \rightarrow \frac{\pi}{2}-\theta_{y}, \delta \rightarrow \delta \pm \pi
$$

we have

$$
\left(M_{\nu}\right)_{i, j}\left(T\left(\theta_{y}\right), T(\delta)\right)=\left(M_{\nu}\right)_{\epsilon(i), \epsilon(j)}\left(\theta_{y}, \delta\right) .
$$

This fact can be, gainfully, used for distinguishing the subcategories of the neutrino mass matrices within the same class.

A remarkable merit of this parametrization is that its three mixing angles $\left(\theta_{x}, \theta_{y}, \theta_{z}\right)$ are directly related to the mixing angles of solar, atmospheric and $\mathrm{CHOOZ}$ reactor neutrino oscillations:

$$
\theta_{x} \approx \theta_{\mathrm{sun}}, \quad \theta_{y} \approx \theta_{\mathrm{atm}}, \quad \theta_{z} \approx \theta_{\mathrm{chz}} .
$$

Also we have,

$$
\Delta m_{\mathrm{sol}}^{2}=\Delta m_{12}^{2}=m_{2}^{2}-m_{1}^{2}, \Delta m_{\text {atm }}^{2}=\left|\Delta m_{23}^{2}\right|=\left|m_{3}^{2}-m_{2}^{2}\right|,
$$

and the hierarchy of solar and atmospheric neutrino mass-squared differences is characterized by the parameter:

$$
R_{\nu} \equiv\left|\frac{m_{2}^{2}-m_{1}^{2}}{m_{3}^{2}-m_{2}^{2}}\right| \approx \frac{\Delta m_{\mathrm{sun}}^{2}}{\Delta m_{\mathrm{atm}}^{2}} \ll 1 .
$$

Reactor nuclear experiments on beta-decay kinematics and neutrinoless double-beta decay put constraints on the neutrino mass scales characterized by the following parameters: the effective electron-neutrino mass

$$
\langle m\rangle_{e}=\sqrt{\sum_{i=1}^{3}\left(\left|V_{e i}\right|^{2} m_{i}^{2}\right)},
$$

and the effective Majorana mass term $\langle m\rangle_{e e}$ given by

$$
\langle m\rangle_{e e}=\left|m_{1} V_{e 1}^{2}+m_{2} V_{e 2}^{2}+m_{3} V_{e 3}^{2}\right| .
$$

Also, cosmological observations put an upper bound on the 'sum' parameter $\Sigma$ which is:

$$
\Sigma=\sum_{i=1}^{3} m_{i} .
$$

A recent global analysis of neutrino experimental data ([17] and references therein), gives the following estimates, at the confidence level of $95 \%$, for the above parameters:

$$
\begin{aligned}
\Delta m_{\mathrm{atm}}^{2} & =\left(2.4_{-0.6}^{+0.5}\right) \times 10^{-3} \mathrm{eV}^{2}, \\
\Delta m_{\mathrm{sol}}^{2} & =(7.92 \pm 0.7) \times 10^{-5} \mathrm{eV}^{2}, \\
\sin ^{2} \theta_{\mathrm{atm}}=\left(0.44_{-0.01}^{+0.18}\right) & \longleftrightarrow \theta_{y}=\left(41.55_{-5.6}^{+10.4}\right) \text { degree }, \\
\sin ^{2} \theta_{\mathrm{sol}}=\left(0.314_{-0.047}^{+0.057}\right) & \longleftrightarrow \theta_{x}=\left(34.08_{-3}^{+3.4}\right) \text { degree } \\
\sin ^{2} \theta_{\mathrm{chz}}=\left(0.9_{-0.9}^{+2.3}\right) \times 10^{-2} & \longleftrightarrow \theta_{z}=\left(5.44_{-5}^{+5}\right) \text { degree}, \\
\langle m\rangle_{e} & <1.8 \mathrm{eV}, \\
\Sigma & <1.4 \mathrm{eV}, \\
\langle m\rangle_{e e} & =\left(0.58_{-0.16}^{+0.22}\right) \mathrm{eV} .
\end{aligned}
$$




\begin{tabular}{|c|c|}
\hline Pattern & Vanishing minors \\
\hline$A_{1}$ & $C_{33}, C_{32}$ \\
$A_{2}$ & $C_{22}, C_{32}$ \\
$B_{3}$ & $C_{33}, C_{31}$ \\
$B_{4}$ & $C_{22}, C_{21}$ \\
$B_{5}$ & $C_{33}, C_{12}$ \\
$B_{6}$ & $C_{22}, C_{13}$ \\
$D$ & $C_{33}, C_{22}$ \\
$S_{1}$ & $C_{31}, C_{11}$ \\
$S_{2}$ & $C_{21}, C_{11}$ \\
$S_{3}$ & $C_{13}, C_{12}$ \\
$F_{1}$ & $C_{33}, C_{11}$ \\
$F_{2}$ & $C_{22}, C_{11}$ \\
$F_{3}$ & $C_{32}, C_{11}$ \\
$F_{4}$ & $C_{31}, C_{32}$ \\
$F_{5}$ & $C_{21}, C_{32}$ \\
\hline
\end{tabular}

Table 1: Vanishing two-minors texture mass matrices.

In particular, the bounds on $R_{\nu}$ put very stringent conditions on any model required to fit the data:

$$
R_{\nu}=\left(0.033_{-0.008}^{+0.016}\right) \text {. }
$$

Note that the lower bound on $\langle m\rangle_{e e}$ disappears if the neutrinoless double-beta decay does not exist. Moreover, this value is obtained based on the 'claimed' observation of [18], which is not unanimously agreed upon.

\section{$3 \quad$ Neutrino mass matrices with two vanishing minors}

As $M_{\nu}$ is $3 \times 3$ symmetric matrix, it totally has 6 independent complex entries, and thus it has 6 independent minors. We will denote by $C_{i j}$ the minor corresponding to the $i j^{t h}$ element (i.e. the determinant of the sub-matrix obtained by deleting the $i^{\text {th }}$ row and the $j^{\text {th }}$ column of $M_{\nu}$ ). Hence, we have 15 possibilities of having vanishing two minors.

Following the classification of $[8,15]$, we list in table 1 the fifteen possible two- vanishing minor texture with its defining minors. The patterns $A_{1}, A_{2}, B_{3}, B_{4}, S_{1}$ and $S_{2}$ are two-texture zeros [15], and the first four of them can accommodate the data [8] (In [8], there were other three acceptable two-texture zero denoted by $B_{1}, B_{2}$ and $C$ which are not reproduced as vanishing two-minor texture). The symmetry $T$ in eqs. $(7,8)$ transforms the neutrino mass matrices of patterns $\left(A_{1}, B_{3}, B_{5}, S_{1}, F_{1}\right.$ and $\left.F_{4}\right)$ to $\left(A_{2}, B_{4}\right.$, $B_{6}, S_{2}, F_{2}$ and $\left.F_{5}\right)$ respectively, whereas the patterns $\left(D, S_{3}\right.$ and $\left.F_{3}\right)$ are singlets under $T$. Therefore, the predictions, for parameters $\left(\theta_{y}, \delta\right)$, of one pattern in the first set are the same as its corresponding pattern in the second set, for parameters $\left(\frac{\pi}{2}-\theta_{y}, \delta \pm \pi\right)$.

If two minors vanish, we have

$$
\begin{aligned}
& M_{\nu a b} M_{\nu c d}-M_{\nu i j} M_{\nu m n}=0, \\
& M_{\nu a^{\prime} b^{\prime}} M_{\nu c^{\prime} d^{\prime}}-M_{\nu i^{\prime} j^{\prime}} M_{\nu m^{\prime} n^{\prime}}=0,
\end{aligned}
$$

then we have

$$
\sum_{l, k=1}^{3}\left(U_{a l} U_{b l} U_{c k} U_{d k}-U_{i l} U_{j l} U_{m k} U_{n k}\right) \lambda_{l} \lambda_{k}=0
$$


and a similar equation with (abcdijmn) replaced by their 'primes'. We get

$$
\begin{aligned}
& \frac{\lambda_{1}}{\lambda_{3}}=\frac{K_{2} L_{1}-K_{1} L_{2}}{K_{2} L_{3}-K_{3} L_{2}} \\
& \frac{\lambda_{2}}{\lambda_{3}}=\frac{K_{2} L_{1}-K_{1} L_{2}}{K_{1} L_{3}-K_{3} L_{1}}
\end{aligned}
$$

where

$$
\begin{aligned}
K_{h} & =\left(U_{a l} U_{b l} U_{c k} U_{d k}-U_{i l} U_{j l} U_{m k} U_{n k}\right)+(l \leftrightarrow k), \\
L_{h} & =\left(U_{a^{\prime} l} U_{b^{\prime} l} U_{c^{\prime} k} U_{d^{\prime} k}-U_{i^{\prime} l} U_{j^{\prime} l} U_{m^{\prime} k} U_{n^{\prime} k}\right)+(l \leftrightarrow k),
\end{aligned}
$$

with $(h, l, k)$ are a cyclic permutation of $(1,2,3)$.

In this way, with the input of four parameters determining the matrix $U$ (the three mixing angles $\theta_{x}$, $\theta_{y}, \theta_{z}$ and the Dirac phase $\delta$ ), we are able to predict the relative magnitude of the three neutrino masses and the values of the two Majorana phases from the relations:

$$
\frac{m_{i}}{m_{3}}=\left|\frac{\lambda_{i}}{\lambda_{3}}\right| \text { for } i=1,2
$$

and

$$
\begin{aligned}
\rho & =\frac{1}{2} \arg \left(\frac{\lambda_{1}}{\lambda_{3}}\right), \\
\sigma & =\frac{1}{2} \arg \left(\frac{\lambda_{2}}{\lambda_{3}}\right) .
\end{aligned}
$$

We can examine now whether or not the chosen texture of $M_{\nu}$ is empirically acceptable by computing the magnitude of the parameter $R_{\nu}$ which should be in the order of $10^{-2}$ (equation 16). With some plausible values of the input parameters, and taking $\Delta m_{\text {sol }}^{2}$ to be its 'central' allowable experimental value, one can reconstruct the mass matrix and test whether or not the other experimental constraints are respected.

We found that the resulting mass patterns could be classified into two categories:

- Normal hierarchy: characterized by $m_{1} \sim m_{2}<m_{3}$ and is denoted by $\mathbf{N}$.

- Inverted hierarchy: characterized by $m_{1} \sim m_{2}>m_{3}$ and is denoted by $\mathbf{I}$.

The possibility of having non-invertible mass matrix is examined for each pattern. The viable noninvertible mass matrices are characterized by vanishing one of the masses $\left(m_{1}\right.$, and $\left.m_{3}\right)$, as compatibility with the data prevents $m_{2}$ to vanish. The conditions and relations satisfied for each possibility are as follows:

- The vanishing of $m_{1}$ implies that $K_{1}=L_{1}=0$ for the same set of parameters, and the mass spectrum of $m_{2}$ and $m_{3}$ takes the values $\sqrt{\Delta m_{\text {sol }}^{2}}$ and $\sqrt{\Delta m_{\text {sol }}^{2}+\Delta m_{\text {atm }}^{2}}$ respectively.

- The vanishing of $m_{3}$ implies that $K_{3}=L_{3}=0$ and the mass spectrum of $m_{2}$ and $m_{1}$ takes the values $\sqrt{\Delta m_{\text {atm }}^{2}}$ and $\sqrt{\Delta m_{\text {atm }}^{2}-\Delta m_{\text {sol }}^{2}}$ respectively.

We present in the following sections the analysis of all the patterns corresponding to two vanishing minors, referred to by their corresponding elements, in the neutrino mass matrix $M_{\nu}$. When the expressions are complicated we only state the analytical leading terms of the expansions in powers of $s_{z}$. The numerical estimates, for quick reference, are shown in table (3), where we fixed, when possible, the input parameters $\left(\theta_{x}, \theta_{y}, \theta_{z}\right)$ to their 'experimental' centered values $\left(34^{\circ}, 42^{\circ}, 5^{\circ}\right)$. 


\section{Class A}

Pattern A1: vanishing minors $\left(C_{33}, C_{32}\right)$ : We get

$$
\begin{aligned}
& \frac{\lambda_{1}}{\lambda_{3}}=\frac{s_{z}}{c_{z}^{2}}\left(\frac{s_{x} s_{y}}{c_{x} c_{y}} e^{i \delta}-s_{z}\right), \\
& \frac{\lambda_{2}}{\lambda_{3}}=-\frac{s_{z}}{c_{z}^{2}}\left(\frac{c_{x} s_{y}}{s_{x} c_{y}} e^{i \delta}+s_{z}\right) .
\end{aligned}
$$

This texture is the two-texture zero neutrino mass matrix having zeros at the $(1,1)$ and $(1,2)$ entries [15]. Consequently, it implies the absence of neutrinoless double-beta decay:

$$
\langle m\rangle_{e e}=0
$$

and thus can have only normal type hierarchy [19]. We have the following analytical approximations:

$$
\begin{aligned}
\frac{m_{1}}{m_{3}} & =t_{x} t_{y} s_{z}-c_{\delta} s_{z}^{2}+O\left(s_{z}^{3}\right), \\
\frac{m_{2}}{m_{3}} & \approx \frac{t_{y}}{t_{x}} s_{z}+c_{\delta} s_{z}^{2}+O\left(s_{z}^{3}\right), \\
\frac{m_{2}}{m_{3}}-\frac{m_{1}}{m_{3}} & =t_{y}\left(\frac{1}{t_{x}}-t_{x}\right) s_{z}+2 c_{\delta} s_{z}^{2}+O\left(s_{z}^{3}\right), \\
R_{\nu} & =\left|\frac{4 t_{y}^{2}}{s_{2 x} t_{2 x}} s_{z}^{2}\right|+O\left(s_{z}^{3}\right), \\
\rho & =\frac{\delta}{2}+\frac{s_{\delta} s_{z}}{2 t_{x} t_{y}}+O\left(s_{z}^{2}\right)\left(\bmod \frac{\pi}{2}\right), \\
\sigma & =\frac{\delta}{2}-\frac{s_{\delta} t_{x} s_{z}}{2 t_{y}}+O\left(s_{z}^{2}\right)\left(\bmod \frac{\pi}{2}\right), \\
\frac{\langle m\rangle_{e}}{m_{3}} & \approx \frac{s_{\delta} s_{z}}{2 t_{y}}\left(t_{x}+\frac{1}{t_{x}}\right)+O\left(s_{z}^{2}\right)\left(\bmod \frac{\pi}{2}\right), \\
c_{y} & s_{z}+O\left(s_{z}^{2}\right) .
\end{aligned}
$$

Taking the constraints eq. (15) into consideration, we see that no quasi-degenerate spectrum can be obtained, since we have $m_{2} / m_{3}<0.4$. We also note that there is a lower bound $\theta_{z}>4.52^{\circ}$, from the expression of $R_{\nu}$ and the maximal and minimal values the parameters can take, and that maximal mixing $\left(\theta_{y}=\frac{\pi}{4}\right)$ is allowed. The parameter $\delta$ is not experimentally constrained and satisfying the $R_{\nu}$-constraint (eq. 16) does not single out specific values for it. We matched the the data with $\left(\theta_{x}=34^{0}, \theta_{y}=\right.$ $\left.42^{0}, \delta=0^{0}, \theta_{z}=7^{0}\right)$. For these inputs we obtain $m_{1} / m_{3}=0.060, m_{2} / m_{3}=0.180, \rho=0^{0}, \sigma=90^{0}$ and $R_{\nu}=0.0298$. The mass $m_{3}$ fitted from the observed $\Delta m_{\mathrm{sol}}^{2}$ is $m_{3}=0.052 \mathrm{eV}$, and the other values for the remaining parameters are $\Delta m_{\mathrm{atm}}^{2}=2.7 \times 10^{-3} \mathrm{eV}^{2},\langle m\rangle_{e}=0.009 \mathrm{eV},\langle m\rangle_{e e}=0 \mathrm{eV}$ and $\Sigma=0.065 \mathrm{eV}$. In this pattern the numerically estimated mass matrix $M$ is

$M_{\nu}=m_{3}\left(\begin{array}{ccc}0.363 \times 10^{-8}-0.228 \times 10^{-10} i & -0.90 \times 10^{-9}-0.234 \times 10^{-10} i & 0.165+0.248 \times 10^{-10} i \\ 0.90 \times 10^{-9}-0.234 \times 10^{-10} i & 0.396-0.241 \times 10^{-10} i & 0.543+0.255 \times 10^{-10} i \\ 0.165+0.248 \times 10^{-10} i & 0.543+0.255 \times 10^{-10} i & 0.483-0.271 \times 10^{-10} i\end{array}\right)$.

Numerically, we find that the estimated mass matrix has a structure of a two-zero texture, as mentioned above, and that we have here a strong hierarchy $m_{2} \ll m_{3}$. The pattern is acceptable, but the numerical fitting, for $\left(\theta_{x}=34^{\circ}, \theta_{y}=42^{\circ}\right)$, is not possible for $\theta_{z}$ quite small, and only for $\theta_{z}$ larger than $7^{\circ}$ 

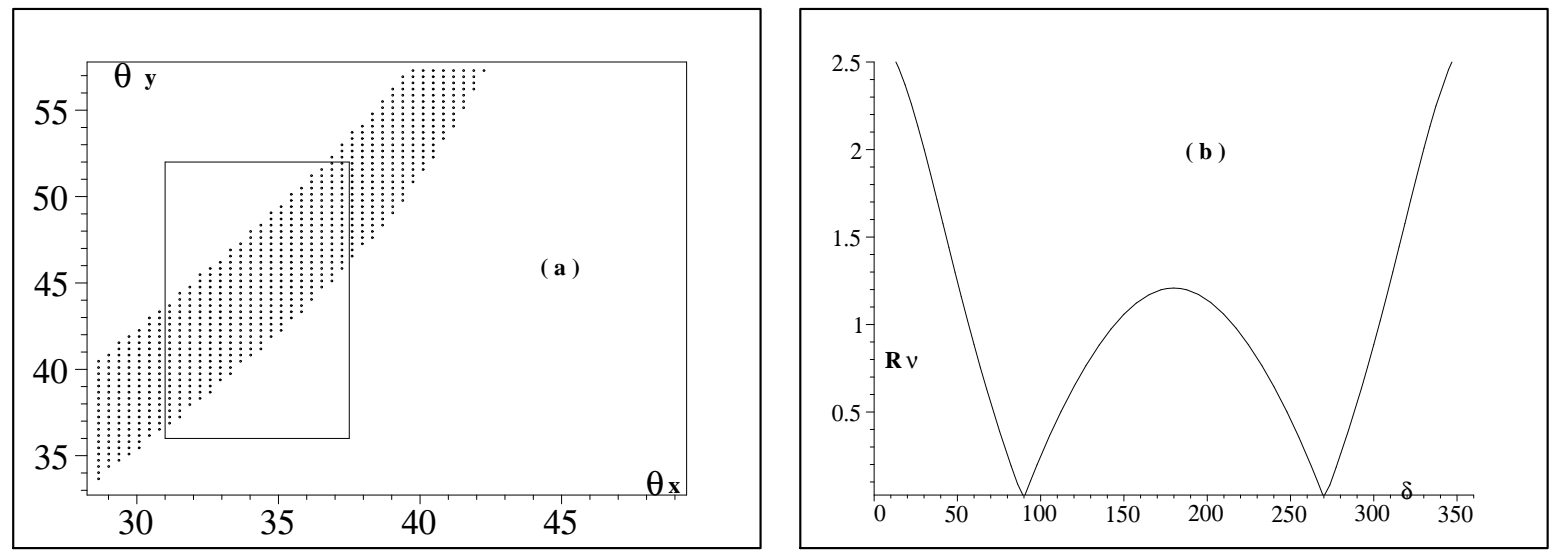

Figure 1: a: The available $\theta_{x}, \theta_{y}$ parameter space for the case $\mathbf{A} 1$ pattern with $\delta=0^{\circ}, \theta_{z}=7^{\circ}$. Both angles $\theta_{x}$ and $\theta_{y}$ are spanned from $29^{0}$ to $57^{0}$. The dots represent the points satisfying all the mass-related constraints, whereas the rectangle delimit the acceptable region for $\theta_{x}$ and $\theta_{y}$. The dots inside the rectangle are the acceptable points. b: $R_{\nu}$ as a function of $\delta$, for $\theta_{x}=34^{\circ}, \theta_{y}=42^{\circ}, \theta_{z}=5^{\circ}$, for case $\mathbf{B 3}$, the values around $\delta=90^{\circ}, 270^{\circ}$ are singled out.

we can accommodate, without tuning, the data. In figure (1.a) we show the parameter space of $\left(\theta_{x}, \theta_{y}\right)$ for $\left(\delta=0^{0}, \theta_{z}=7^{0}\right)$ where the rectangle delimit the experimentally acceptable region.

One can show analytically that an acceptable phenomenology for this two-vanishing minors texture would exclude the non-invertible mass matrix possibility and would lead automatically to a two-zero entries texture. In fact, the conditions in eq. (17) and eq.(18) reading now as:

$$
\begin{aligned}
& M_{\nu 11} M_{\nu 22}-M_{\nu 12} M_{\nu 21}=0 \quad\left(C_{33}=0\right), \\
& M_{\nu 11} M_{\nu 23}-M_{\nu 13} M_{\nu 21}=0 \quad\left(C_{32}=0\right),
\end{aligned}
$$

will imply, with a non-zero $M_{\nu 11}$ or $M_{\nu 12}$, the vanishing of the the minor

$$
C_{31}=M_{\nu 12} M_{\nu 23}-M_{\nu 13} M_{\nu 22}
$$

which, together with $C_{33}=C_{32}=0$, means that the mass matrix is singular. Actually, a non-zero $M_{\nu 11}$, say, leads to either $M_{\nu 22}=0$, and hence, from eq. 31, to $M_{\nu 21}=0$ implying the vanishing of $C_{31}$, or to $M_{\nu 22} \neq 0$ and so equation 31 leads to

$$
M_{\nu 11}=\frac{M_{\nu 12} M_{\nu 12}}{M_{\nu 22}} .
$$

In the latter case $\left(M_{\nu 22} \neq 0\right)$, we have two possibilities. The first is that $M_{\nu 23}=0$ which, via eq. 32 , leads to either $M_{\nu 13}=0$ and hence $C_{31}=0$, or $M_{\nu 12}=0$ but then, from eq. 34, we have a vanishing $M_{\nu 11}$. The second possibility is that $M_{\nu 23} \neq 0$ and hence we have (see eq. 32)

$$
M_{\nu 11}=\frac{M_{\nu 13} M_{\nu 21}}{M_{\nu 23}}
$$


The two equations (34 and 35) imply again either a vanishing $\left(M_{\nu 21}\right.$ and $\left.M_{\nu 11}\right)$ or the minor $C_{31}$ equals to zero. We could check also that a non-zero $M_{\nu 12}$ leads again to a vanishing-determinant matrix. However, a consistent, phenomenologically acceptable, solution with one of the masses $m_{1}$ or $m_{3}$ equal to zero, could not be found. Actually, the vanishing of $m_{3}$ in this texture leads to:

$$
\begin{aligned}
K_{3} & =c_{z}^{2} c_{y}^{2} e^{-2 i \delta}=0 \quad\left(\text { following from } C_{33}\right) \\
L_{3} & =-c_{z}^{2} c_{y} s_{y} e^{-2 i \delta}=0 \quad\left(\text { following from } C_{32}\right)
\end{aligned}
$$

which is satisfied by the non-admissible values $\theta_{z}$ or $\theta_{y}$ equal to $\frac{\pi}{2}$.

As to the vanishing of $m_{1}$, it leads to more involved expressions::

$$
\begin{aligned}
K_{1} & \left.=\left(-s_{x} s_{y}+s_{z} c_{x} c_{y} e^{-i \delta}\right)^{2}=0 \quad \text { (following from } C_{33}\right), \\
L_{1} & \left.=\left(s_{x} c_{y}+s_{z} c_{x} s_{y} e^{-i \delta}\right)\left(s_{x} s_{y}-s_{z} c_{x} c_{y} e^{-i \delta}\right)=0 \quad \text { (following from } C_{32}\right),
\end{aligned}
$$

These two conditions are met if and only if $\left(s_{x} s_{y}-s_{z} c_{x} c_{y} e^{-i \delta}\right)=0$. However, putting the imaginary part of the last expression equal to zero, we see directly, since $c_{x} c_{y} \neq 0$, that $s_{\delta}=0$ otherwise $s_{z}=0$ implying the phenomenologically rejected possibility $s_{x} s_{y}=0$. Now, with the angles $\theta_{x}, \theta_{y}, \theta_{z}$ being in the first quarter, we should have $\delta=0$ leading to

$$
t_{x}=\frac{s_{z}}{t_{y}}
$$

One can easily check that for acceptable choices of the parameters $\theta_{x}, \theta_{y}$ and $\theta_{z}$ the above equation can not be satisfied.

Pattern A2: vanishing minors $\left(C_{22}, C_{32}\right)$ : We get

The analytical expressions, and the representative numerical results, of the pattern $\mathbf{A} \mathbf{1}$ are valid here after the substitution dictated by eqs. $(7,8):\left(c_{y} \leftrightarrow s_{y}, c_{\delta} \rightarrow-c_{\delta}, s_{\delta} \rightarrow-s_{\delta}\right)$ and interchanging the mass matrix indices $(2 \leftrightarrow 3)$. The conclusions stay the same, but with a different lower bound $\theta_{z}>4.25^{\circ}$. As to the parameter space of $\left(\theta_{x}, \theta_{y}\right)$, one should do a symmetry with respect to the line $\left(\theta_{y}=45^{\circ}\right)$ in figure 1.a to get the parameter space in this pattern. It would be difficult to distinguish experimentally between the two patterns in class $\mathrm{A}$.

\section{Class B}

Pattern B3: vanishing minors $\left(C_{33}, C_{31}\right)$ : We get

$$
\begin{aligned}
& \frac{\lambda_{1}}{\lambda_{3}}=-\frac{s_{y}}{c_{y}} \cdot \frac{s_{x} s_{y}-c_{x} c_{y} s_{z} e^{-i \delta}}{s_{x} c_{y}+c_{x} s_{y} s_{z} e^{i \delta}} e^{2 i \delta}, \\
& \frac{\lambda_{2}}{\lambda_{3}}=-\frac{s_{y}}{c_{y}} \cdot \frac{c_{x} s_{y}+s_{x} c_{y} s_{z} e^{-i \delta}}{c_{x} c_{y}-s_{x} s_{y} s_{z} e^{i \delta}} e^{2 i \delta} .
\end{aligned}
$$

We have the following analytical approximations:

$$
\begin{aligned}
\frac{m_{1}}{m_{3}} & =t_{y}^{2}-\frac{s_{y} c_{\delta} s_{z}}{t_{x} c_{y}^{3}}+O\left(s_{z}^{2}\right), \\
\frac{m_{2}}{m_{3}} & =t_{y}^{2}+\frac{t_{x} s_{y} c_{\delta} s_{z}}{c_{y}^{3}}+O\left(s_{z}^{2}\right), \\
\frac{m_{2}}{m_{3}}-\frac{m_{1}}{m_{3}} & =\frac{s_{y} c_{\delta} s_{z}}{c_{y}^{3}}\left(t_{x}+\frac{1}{t_{x}}\right)+O\left(s_{z}^{2}\right), \\
R_{\nu} & \approx\left|\frac{1+t_{x}^{2}}{t_{x}} t_{y}^{2} t_{2 y} c_{\delta} s_{z}\right|+O\left(s_{z}^{2}\right),
\end{aligned}
$$




$$
\begin{aligned}
\rho & =\delta+\frac{s_{\delta} s_{z}}{t_{2 y} t_{x}}+O\left(s_{z}\right)\left(\bmod \frac{\pi}{2}\right), \\
\sigma & =\delta-\frac{t_{x} s_{\delta} s_{z}}{t_{2 y}}+O\left(s_{z}\right)\left(\bmod \frac{\pi}{2}\right), \\
\rho-\sigma & =\frac{s_{\delta} s_{z}}{t_{2 y}}\left(t_{x}+\frac{1}{t_{x}}\right)+O\left(s_{z}\right)\left(\bmod \frac{\pi}{2}\right), \\
\frac{\langle m\rangle_{e e}}{m_{3}} & \approx t_{y}^{2}+O\left(s_{z}\right), \\
\frac{\langle m\rangle_{e}}{m_{3}} & \approx t_{y}^{2}+O\left(s_{z}\right) .
\end{aligned}
$$

In order that $m_{2}$ be larger than $m_{1}$, we see that the parameter $\delta$ is restricted to be in the first and fourth quadrants. We see also that N-type (I-type) -hierarchy can be obtained if $\theta_{y}<\frac{\pi}{4}\left(\theta_{y}>\frac{\pi}{4}\right)$. For $\theta_{z}$ not too small, satisfying the $R_{\nu}$-constraint singles out the right angles $\left(\frac{\pi}{2}, \frac{3 \pi}{2}\right)$ for $\delta$, as can be seen in figure (1.b). When we approach the maximal mixing limit $\left(\theta_{y}=\frac{\pi}{4}\right)$ then, from the $R_{\nu}$ expression, $\theta_{z}$ tends to zero or $\delta$ tends to a right angle. However, from eq. 39, the limit is not attained since $\left(\theta_{y}=\frac{\pi}{4}\right)$ leads to a degenerate spectrum $\left(m_{1}=m_{2}=m_{3}\right)$. No lower bounds on $\theta_{z}$ can be obtained.

For the N-type, we take the representative point $\left(\theta_{x}=34^{0}, \theta_{y}=42^{0}, \theta_{z}=5^{0}\right)$, and find that the $R_{\nu}$ condition constrains the Dirac phase to be around $\delta=88.3^{\circ}$. For these inputs we obtain $m_{1} / m_{3}=$ $0.8073998611, m_{2} / m_{3}=0.8141569633, \rho=179.093^{0}, \sigma=177.953^{\circ}$ and $R_{\nu}=0.0325$. The mass $m_{3}$ fitted from the observed $\Delta m_{\mathrm{sol}}^{2}$ is then $m_{3}=0.085 \mathrm{eV}$, whereas the values for the other remaining parameters would be $\Delta m_{\text {atm }}^{2}=2.5 \times 10^{-3} \mathrm{eV}^{2},\langle m\rangle_{e}=0.069 \mathrm{eV},\langle m\rangle_{e e}=0.069 \mathrm{eV}$ and $\Sigma=0.223 \mathrm{eV}$. The numerically estimated mass matrix $M$ is

$$
M_{\nu}=m_{3}\left(\begin{array}{ccc}
0.810-0.0355 i & -0.213 \times 10^{-8}+0.125 \times 10^{-7} i & 0.022+0.417 \times 10^{-2} i \\
-0.213 \times 10^{-8}+0.125 \times 10^{-7} i & -0.99 \times 10^{-8}-0.202 \times 10^{-8} i & 0.900+0.334 \times 10^{-9} i \\
0.022+0.417 \times 10^{-2} i & 0.900+0.334 \times 10^{-9} i & 0.187-0.491 \times 10^{-3} i
\end{array}\right)
$$

For the choice $\delta=88.3^{\circ}$ and $\theta_{z}=5^{\circ}$, the acceptable region in the parameter space of $\theta_{x}, \theta_{y}$ is depicted in figure (2.a) which indicates that no tuning is required for the mixing angles $\theta_{x}$ and $\theta_{y}$ to assure their consistency with the data.

For the I-type hierarchy, we take the representative point $\left(\theta_{x}=34^{0}, \theta_{y}=48^{0}, \theta_{z}=5^{0}\right)$, then the $R_{\nu^{-}}$ constraint singles out the value $\delta=270.4^{\circ}$. For these inputs we obtain $m_{1} / m_{3}=1.226998357, m_{2} / m_{3}=$ $1.233576775, \rho=1.16^{0}, \sigma=0.05^{0}$ and $R_{\nu}=0.031$. The mass $m_{3}$ fitted from the observed $\Delta m_{\text {sol }}^{2}$ is $m_{3}=0.07 \mathrm{eV}$. Then the derived values for the other remaining parameters are $\Delta m_{\mathrm{atm}}^{2}=2.6 \times 10^{-3} \mathrm{eV}^{2}$, $\langle m\rangle_{e}=0.086 \mathrm{eV},\langle m\rangle_{e e}=0.086 \mathrm{eV}$ and $\Sigma=0.242 \mathrm{eV}$. In this I-type case, the numerically estimated mass matrix $M$ is

$$
M_{\nu}=m_{3}\left(\begin{array}{ccc}
1.227+0.034 i & -0.35 \times 10^{-9}+0.6 \times 10^{-9} i & -0.030-0.005 i \\
-0.35 \times 10^{-9}+0.6 \times 10^{-9} i & -0.33 \times 10^{-8}-6.0 \times 10^{-11} i & 1.111+2.247 \times 10^{-12} i \\
-0.030-0.005 i & 1.111+2.247 \times 10^{-12} i & -0.226+0.001 i
\end{array}\right)
$$

The parameter space of $\theta_{x}, \theta_{y}$, for the choice $\delta=270.4^{\circ}$ and $\theta_{z}=5^{\circ}$, is depicted in figure (2.b). We see that it is quite large and no tuning is necessary.

The numerical solutions in both types of hierarchy reproduce a two-zero texture, which can accommodate acceptably the data. As in class A, one could show analytically that a two-vanishing minors such texture with non-vanishing entries leads to a singular mass matrix. Nevertheless, as in class A, no consistent solution could be found when attaining the limit of one vanishing mass. 

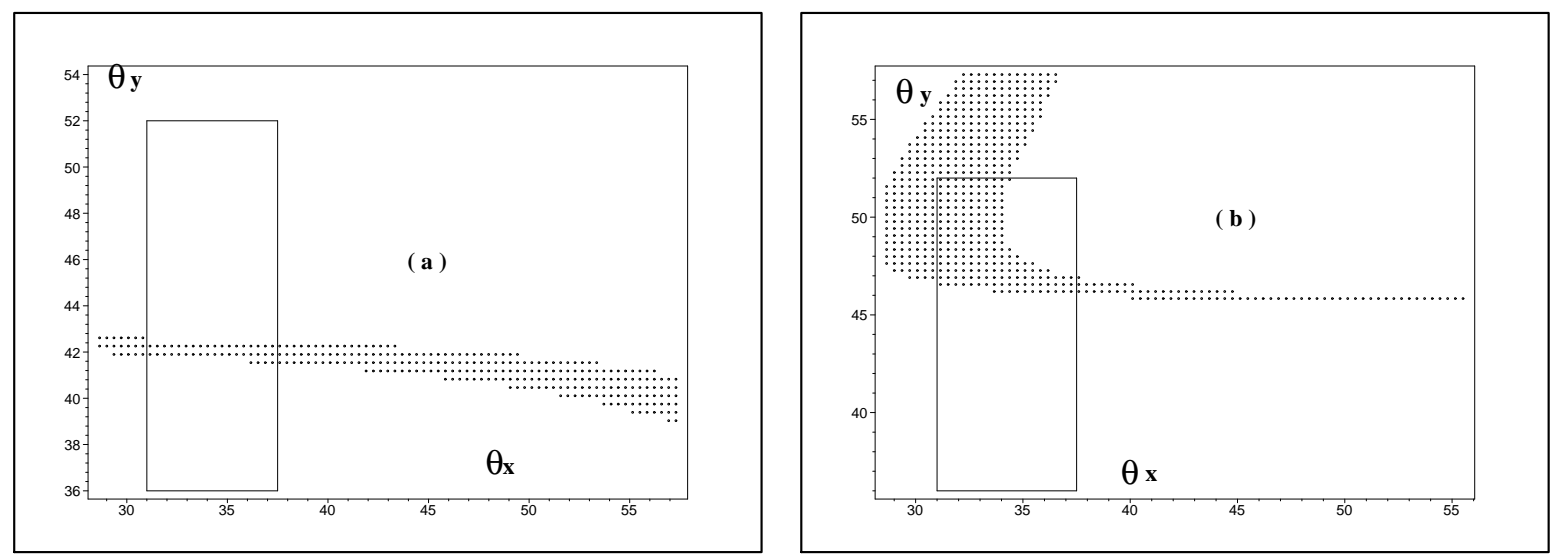

Figure 2: The available $\theta_{x}, \theta_{y}$ parameter space for the B3 pattern (a) N-type hierarchy with $\delta=88.3^{\circ}$ and (b) I-type hierarchy with $\delta=270.4^{\circ}$. The range from $29^{0}$ to $57^{\circ}$ is spanned for both angles $\theta_{x}$ and $\theta_{y}$. The dots represent the set of points $\left(\theta_{x}, \theta_{y}\right)$ which satisfy all the mass-related constraints. The rectangle is the acceptable region for $\theta_{x}, \theta_{y}$. The points inside the rectangle are the acceptable points.

Pattern B4: vanishing minors $\left(C_{22}, C_{21}\right)$ :

The analytical expressions, and the representative numerical results, of the pattern $\mathbf{B} 3$ are valid here after the substitution prescribed by eqs. $(7,8)$. The conclusions are similar in that $\delta$ is now restricted to the second and third quadrants, and that we have N-type (I-type) hierarchy when $\theta_{y}$ is larger (smaller) than $\frac{\pi}{4}$. The parameter space of $\left(\theta_{x}, \theta_{y}\right)$, depicted in figure (2.b), should be reflected through the line $\left(\theta_{y}=45^{\circ}\right)$ to get what corresponds $\mathbf{B} 4$-pattern.

Pattern B5: vanishing minors $\left(C_{33}, C_{12}\right)$ : We get

$$
\begin{aligned}
\frac{\lambda_{1}}{\lambda_{3}} & =\frac{s_{x} c_{x} c_{y} s_{z}^{2}\left(1+s_{y}^{2}\right) e^{-2 i \delta}+s_{z} s_{y}^{3} c_{2 x} e^{-i \delta}+s_{x} c_{y} c_{x} s_{y}^{2}}{s_{z} s_{y} c_{y}^{2} s_{x}^{2} e^{-3 i \delta}+\left[-c_{x} c_{y}^{3} s_{x}\left(1+s_{z}^{2}\right)+2 s_{x} c_{y} c_{x} s_{z}^{2}\right] e^{-2 i \delta}+s_{z} c_{x}^{2} s_{y}^{3} e^{-i \delta}} \\
\frac{\lambda_{2}}{\lambda_{3}} & =\frac{-s_{x} c_{y} c_{x} s_{z}^{2}\left(1+s_{y}^{2}\right) e^{-2 i \delta}-s_{y}^{3} s_{z} c_{2 x} e^{-i \delta}-c_{x} s_{x} c_{y} s_{y}^{2}}{s_{z} s_{y} c_{y}^{2} c_{x}^{2} e^{-3 i \delta}+\left[-2 s_{x} c_{x} c_{y} s_{z}^{2}+s_{x} c_{x} c_{y}^{3}\left(1+s_{z}^{2}\right)\right] e^{-2 i \delta}+s_{z} s_{x}^{2} s_{y}^{3} e^{-i \delta}}
\end{aligned}
$$

We have the following analytical approximations:

$$
\begin{aligned}
\frac{m_{1}}{m_{3}} & =t_{y}^{2}+\frac{t_{y}^{3} c_{\delta} s_{z}}{c_{y}^{2} t_{x}}+O\left(s_{z}^{2}\right), \\
\frac{m_{2}}{m_{3}} & =t_{y}^{2}-\frac{t_{y}^{3} c_{\delta} t_{x} s_{z}}{c_{y}^{2}}+O\left(s_{z}^{2}\right), \\
\frac{m_{2}}{m_{3}}-\frac{m_{1}}{m_{3}} & =-\frac{c_{\delta} t_{y}^{3} s_{z}}{c_{y}^{2}}\left(t_{x}+\frac{1}{t_{x}}\right)++O\left(s_{z}^{2}\right), \\
R_{\nu} & =\left|\frac{-4 s_{y}^{2} t_{y}^{3} c_{\delta}}{c_{2 x} c_{2 y}} s_{z}\right|+O\left(s_{z}^{2}\right)
\end{aligned}
$$




$$
\begin{aligned}
\rho & =\delta-\frac{t_{x} c_{2 y} s_{\delta}}{2 c_{y}^{2} t_{y}} s_{z}+O\left(s_{z}^{2}\right)\left(\bmod \frac{\pi}{2}\right), \\
\sigma & =\delta+\frac{c_{2 y} t_{y} s_{\delta}}{2 c_{y}^{2} t_{x}} s_{z}+O\left(s_{z}^{2}\right)\left(\bmod \frac{\pi}{2}\right), \\
\rho-\sigma & =-\frac{c_{2 y} s_{\delta}}{2 c_{y}^{2}}\left(\frac{t_{x}}{t_{y}}+\frac{t_{y}}{t_{x}}\right) s_{z}+O\left(s_{z}^{2}\right)\left(\bmod \frac{\pi}{2}\right), \\
\frac{\langle m\rangle_{e e}}{m_{3}} & =t_{y}^{2}+O\left(s_{z}\right), \\
\frac{\langle m\rangle_{e}}{m_{3}} & =t_{y}^{2}+O\left(s_{z}\right) .
\end{aligned}
$$

This pattern is not a two-texture zero [15], and the parameter $\delta$ is restricted to be in the second and third quadrants. The N-type (I-type) -hierarchy can be obtained if $\theta_{y}<\frac{\pi}{4}\left(\theta_{y}>\frac{\pi}{4}\right)$. We see that, for $\theta_{z}$ not too small, the angle $\delta$ needs to be peaked around a right angle in order to satisfy the $R_{\nu^{-}}$ constraint. The maximal mixing limit can not be reached since $\left(\theta_{y}=\frac{\pi}{4}\right)$ leads to a degenerate spectrum $\left(m_{1}=m_{2}=m_{3}\right)$. However, when $\left(\theta_{y} \rightarrow \frac{\pi}{4}\right)$ then $\theta_{z}$ tends to zero or $\delta$ tends to a right angle. No lower bounds on $\theta_{z}$ can be obtained.

For the N-type, we take the representative point $\left(\theta_{x}=34^{0}, \theta_{y}=42^{0}, \theta_{z}=5^{0}\right)$, and the the $R_{\nu^{-}}$ condition constrains $\delta$ to be around $92^{\circ}$. For these inputs we obtain $m_{1} / m_{3}=0.8018004128, m_{2} / m_{3}=$ $0.8090251390, \rho=1.4^{0}, \sigma=2.34^{0}$ and $R_{\nu}=0.0336$. The mass $m_{3}$ fitted from the observed $\Delta m_{\text {sol }}^{2}$ is $m_{3}=0.082 \mathrm{eV}$, and then the values for the other remaining parameters are inferred to be $\Delta m_{\mathrm{atm}}^{2}=$ $2.4 \times 10^{-3} \mathrm{eV}^{2},\langle m\rangle_{e}=0.066 \mathrm{eV},\langle m\rangle_{e e}=0.066 \mathrm{eV}$ and $\Sigma=0.215 \mathrm{eV}$. The numerically estimated mass matrix $M$, in this pattern, is

$$
M_{\nu}=m_{3}\left(\begin{array}{ccc}
0.804+0.047 i & 0.021-0.495 \times 10^{-2} i & 0.441 \times 10^{-2}-0.107 \times 10^{-2} i \\
0.021-0.495 \times 10^{-2} i & 0.487 \times 10^{-3}-0.284 \times 10^{-3} i & 0.898+0.839 \times 10^{-3} i \\
0.441 \times 10^{-2}-0.107 \times 10^{-2} & 0.898+0.839 \times 10^{-3} i & 0.191-0.629 \times 10^{-3} i
\end{array}\right) .
$$

For the choice $\delta=92^{\circ}$ and $\theta_{z}=5^{\circ}$, the acceptable region in the parameter space of $\theta_{x}, \theta_{y}$ is quite large and is depicted in figure (3.a), thus no tuning is necessary for the mixing angles $\theta_{x}$ and $\theta_{y}$ to assure their consistency with the data.

For the I-type hierarchy, we take the representative point $\left(\theta_{x}=34^{0}, \theta_{y}=48^{0}, \theta_{z}=5^{0}\right)$, then the $R_{\nu}$-constraint singles out the value $\delta=269.83^{\circ}$. For these inputs we obtain $m_{1} / m_{3}=1.234417996$, $m_{2} / m_{3}=1.241674367, \rho=178.84^{\circ}, \sigma=0.26^{\circ}$ and $R_{\nu}=0.033$. The mass $m_{3}$ fitted from the observed $\Delta m_{\text {sol }}^{2}$ is $m_{3}=0.066 \mathrm{eV}$. Then the derived values for the other remaining parameters are $\Delta m_{\text {atm }}^{2}=$ $2.4 \times 10^{-3} \mathrm{eV}^{2},\langle m\rangle_{e}=0.082 \mathrm{eV},\langle m\rangle_{e e}=0.082 \mathrm{eV}$ and $\Sigma=0.231 \mathrm{eV}$. In this I-type case, the numerically estimated mass matrix $M$ is

$$
M_{\nu}=m_{3}\left(\begin{array}{ccc}
1.234-0.03055 i & -0.03414+0.004469 i & 0.007299-0.0009687 i \\
-0.03414+0.004469 i & 0.0009338-0.0002241 i & 1.114-0.0003354 i \\
0.007299-0.0009687 i & 1.114-0.0003354 i & -0.2382+0.0004991 i
\end{array}\right)
$$

The parameter space of $\theta_{x}, \theta_{y}$, for the choice $\delta=269.83^{\circ}$ and $\theta_{z}=5^{\circ}$, is depicted in figure (3.b). Again it is quite large and no tuning is necessary.

The numerical solutions in both types of hierarchy are not two-zero textures. The pattern is acceptable. However, it does not allow for non-invertible mass matrices as no consistent solution could be found in the limit of one vanishing mass.

Pattern B6: vanishing minors $\left(C_{22}, C_{31}\right)$ : 

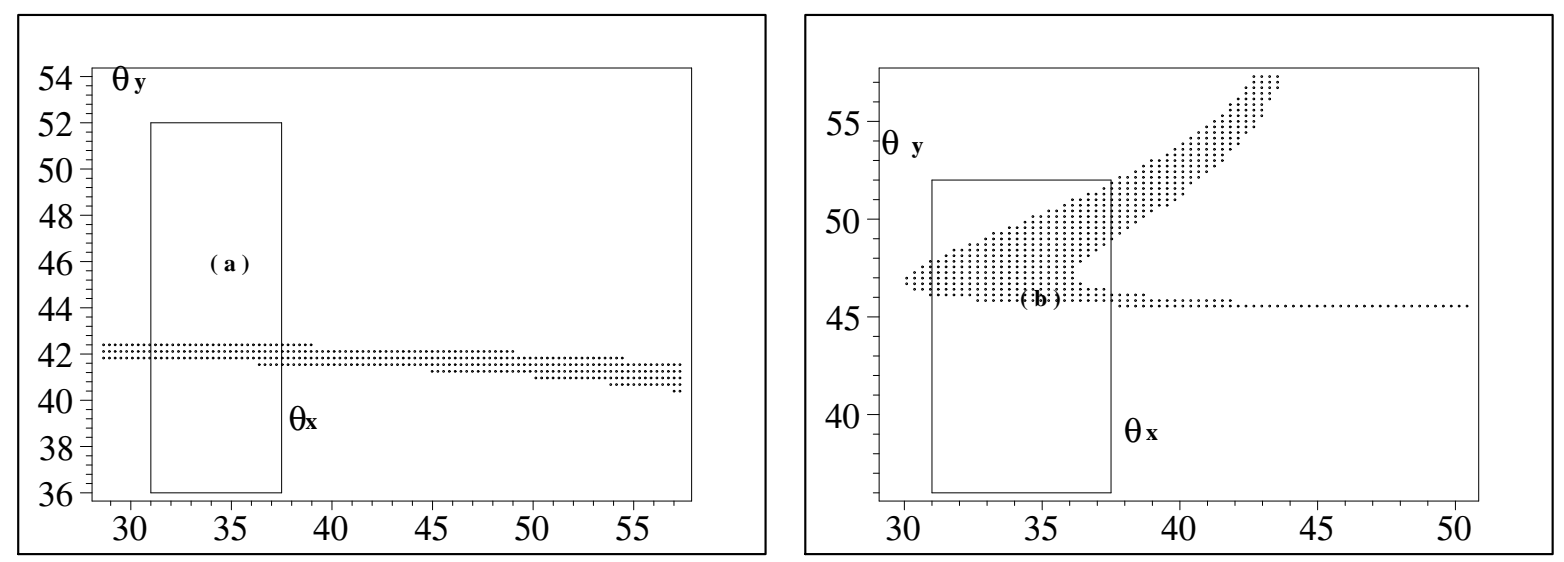

Figure 3: The available $\theta_{x}, \theta_{y}$ parameter space for the $\mathbf{B 5}$ pattern (a) N-type hierarchy with $\delta=92^{\circ}$ and (b) I-type hierarchy with $\delta=269.83^{\circ}$. The range from $29^{0}$ to $57^{0}$ is spanned for both angles $\theta_{x}$ and $\theta_{y}$. The dots represent the set of points $\left(\theta_{x}, \theta_{y}\right)$ which satisfy all the mass-related constraints. The rectangle is the acceptable region for $\theta_{x}, \theta_{y}$. The points inside the rectangle are the acceptable points.

The analytical expressions, and the representative numerical results, of the pattern B5 are valid here after the substitution imposed by eqs. $(7,8)$. The conclusions are similar in that $\delta$ is now restricted to the first and fourth quadrants, and that we have N-type (I-type) hierarchy when $\theta_{y}$ is larger (smaller) than $\frac{\pi}{4}$. The parameter space of $\left(\theta_{x}, \theta_{y}\right)$, depicted in figure (3.b), should be reflected through the line $\left(\theta_{y}=45^{\circ}\right)$ to get what corresponds $\mathbf{B} 6$-pattern.

The class (B) can be distinguished easily from class (A) in that it allows for I-type hierarchy. We summarize in table (2) the 'experimental' signatures which enable to distinguish between its patterns.

\begin{tabular}{|c||c|c|c|}
\hline Model & $\delta$-Quadrant & I-type & N-type \\
\hline \hline B3 & 1,4 & $\theta_{y}>\frac{\pi}{4}$ & $\theta_{y}<\frac{\pi}{4}$ \\
\hline B4 & 2,3 & $\theta_{y}<\frac{\pi}{4}$ & $\theta_{y}>\frac{\pi}{4}$ \\
\hline B5 & 2,3 & $\theta_{y}>\frac{\pi}{4}$ & $\theta_{y}<\frac{\pi}{4}$ \\
\hline B6 & 1,4 & $\theta_{y}<\frac{\pi}{4}$ & $\theta_{y}>\frac{\pi}{4}$ \\
\hline
\end{tabular}

Table 2: 'Experimental' Signatures distinguishing the different B-class patterns.

\section{Class D}

Pattern D: vanishing minors $\left(C_{33}, C_{22}\right)$ : We get

$$
\frac{\lambda_{1}}{\lambda_{3}}=\frac{-2 c_{x} s_{x} s_{y} c_{y} s_{z}^{3} e^{-2 i \delta}+\left(-s_{z}^{2} c_{2 y}+2 c_{x}^{2} s_{z}^{2} c_{2 y}\right) e^{-i \delta}-2 s_{x} c_{x} s_{y} c_{y} s_{z}}{c_{z}^{2} c_{x}\left(-c_{x} c_{2 y}+2 s_{x} c_{y} s_{z} s_{y} e^{-i \delta}\right) e^{-i \delta}}
$$




$$
\frac{\lambda_{2}}{\lambda_{3}}=\frac{-2 c_{x} s_{x} s_{y} c_{y} s_{z}^{3} e^{-2 i \delta}+\left(-s_{z}^{2} c_{2 y}+2 c_{x}^{2} s_{z}^{2} c_{2 y}\right) e^{-i \delta}-2 s_{x} c_{x} s_{y} c_{y} s_{z}}{c_{z}^{2}\left(s_{x}^{2} c_{2 y}+2 s_{x} c_{y} s_{z} c_{x} s_{y} e^{-i \delta}\right) e^{-i \delta}}
$$

We have the following analytical approximations:

$$
\begin{aligned}
\frac{m_{1}}{m_{3}} & =t_{x}\left|t_{2 y}\right| s_{z}+O\left(s_{z}^{2}\right), \\
\frac{m_{2}}{m_{3}} & =\frac{\left|t_{2 y}\right|}{t_{x}} s_{z}+O\left(s_{z}^{2}\right), \\
\frac{m_{2}}{m_{3}}-\frac{m_{1}}{m_{3}} & =\left|t_{2 y}\right|\left(\frac{1}{t_{x}}-t_{x}\right) s_{z}+O\left(s_{z}^{2}\right), \\
R_{\nu} & =\frac{4 t_{2 y}^{2} s_{z}^{2}}{s_{2 x} t_{2 x}}+O\left(s_{z}^{3}\right) \approx \frac{4 t_{x}^{2}\left(\frac{m_{2}}{m_{3}}\right)^{2}}{s_{2 x} t_{2 x}}+O\left(s_{z}^{3}\right), \\
\rho & =\frac{\delta}{2}+2 \frac{c_{2 x}-c_{x}^{2} s_{2 y}^{2}}{s_{2 x} s_{4 y}} s_{\delta} s_{z}+O\left(s_{z}^{2}\right)\left(\bmod \frac{\pi}{2}\right), \\
\sigma & =\frac{\delta}{2}+2 \frac{c_{2 x}+s_{x}^{2} s_{2 y}^{2}}{s_{2 x} s_{4 y}} s_{\delta} s_{z}+O\left(s_{z}^{2}\right)\left(\bmod \frac{\pi}{2}\right), \\
\rho-\sigma & =-\frac{t_{2 y}}{s_{2 x}} s_{\delta} s_{z}+O\left(s_{z}^{2}\right)\left(\bmod \frac{\pi}{2}\right), \\
\frac{\langle m\rangle_{e e}}{m_{3}} & =\frac{1}{c_{2 y}^{2}} s_{z}^{2}+O\left(s_{z}^{3}\right), \\
\frac{\left\langle m_{e}\right.}{m_{3}} & =\frac{1}{c_{2 y}} s_{z}+O\left(s_{z}^{2}\right) .
\end{aligned}
$$

We see that one can not obtain an I-type hierarchy and satisfy the $R_{\nu}$-constraint simultaneously. Although the first term in the expansion of $R_{\nu}$ in powers of $s_{z}$ is independent of $\delta$, we find numerically that satisfying the $R_{\nu}$-constraint, for fixed $\left(\theta_{x}, \theta_{y}\right.$ and $\left.\theta_{z}\right)$, singles out specific values of $\delta$. In fact, upon closer examination, we found that the next term in the expansion to be of the same order of the first term, and there is a delicate cancelation enforcing the small value of $R_{\nu}$ :

$$
R_{\nu}=\left|\frac{4 t_{2 y}^{2} s_{z}^{2}}{s_{2 x} t_{2 x}}-\frac{2 c_{\delta} t_{2 y} s_{z}^{3}}{s_{x}^{3} c_{x}^{3} c_{2 y}^{2}}\left[1-s_{2 x}^{2}\left(1-\frac{1}{4} s_{2 y}^{2}\right)\right]\right|+O\left(s_{z}^{4}\right),
$$

and so for plausible values of $\theta_{x}$ and $\theta_{y}$ the expansion parameter $s_{z}$ might not be the right one. Moreover, eq.(49) tells us that although $\delta$ is not constrained, however it is correlated to the angle $\theta_{y}$ in that when the latter is smaller than $\frac{\pi}{4}$ the angle $\delta$ needs to be in the first or fourth quadrants $\left(c_{\delta}>0\right)$, whereas $\theta_{y}$ being larger than $\frac{\pi}{4}$ makes the angle $\delta$ to be in the second and third quadrants $\left(c_{\delta}<0\right)$. Also, there is no lower bounds on $s_{z}$, however, for $s_{z}$ small enough the angle $\theta_{y}$ approaches the maximal mixing limit.

As a representative point for this pattern we take $\left(\theta_{x}=34^{0}, \theta_{y}=41^{0}, \theta_{z}=5^{0}\right)$, and find that the $R_{\nu}$-constraint singles out the value $\delta=52.7^{\circ}$. For these inputs we obtain $m_{1} / m_{3}=0.5115705296$, $m_{2} / m_{3}=0.5342013580, \rho=14.35^{\circ}, \sigma=128.95^{\circ}$ and $R_{\nu}=0.033$. The mass $m_{3}$ fitted from the observed $\Delta m_{\mathrm{sol}}^{2}$ is $m_{3}=0.0578 \mathrm{eV}$. Then the derived values for the other remaining parameters are $\Delta m_{\mathrm{atm}}^{2}=2.40 \times 10^{-3} \mathrm{eV}^{2},\langle m\rangle_{e}=0.030 \mathrm{eV},\langle m\rangle_{e e}=0.016 \mathrm{eV}$ and $\Sigma=0.118 \mathrm{eV}$. In this pattern the numerically estimated mass matrix $M$ is

$$
M_{\nu}=m_{3}\left(\begin{array}{ccc}
0.279+0.006 i & -0.290-0.007 i & 0.336+0.006 i \\
-0.290-0.007 i & 0.302+0.009 i & 0.641-0.007 i \\
0.336+0.006 i & 0.641-0.007 i & 0.404+0.005 i
\end{array}\right) .
$$


The parameter space of $\theta_{x}, \theta_{y}$, for the choice $\delta=59.3^{\circ}$ and $\theta_{z}=5^{\circ}$, is depicted in figure (4). We found no consistent non-invertible such texture which can accommodate the data.

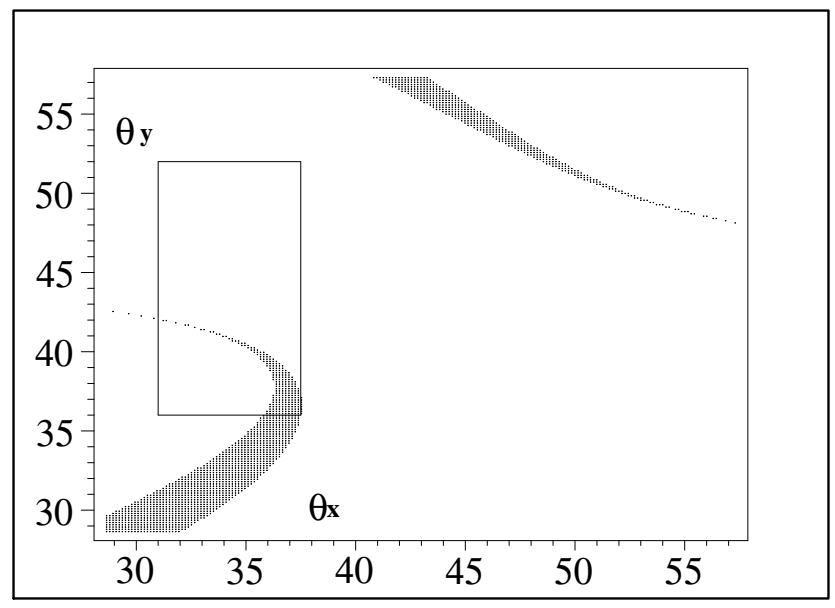

Figure 4: The available $\theta_{x}, \theta_{y}$ parameter space for the $\mathbf{D}$ pattern with $\delta=52.7^{\circ}$. The hierarchy is of $\mathrm{N}$-type, and we have spanned both $\theta_{x}$ and $\theta_{y}$ from $29^{0}$ to $57^{0}$. The dots represent the set of points $\left(\theta_{x}, \theta_{y}\right)$ which satisfy all the mass-related constraints, whereas the rectangle is the acceptable region for $\theta_{x}, \theta_{y}$. The points inside the rectangle are the acceptable points.

This class is hardly distinguishable from the class $\mathbf{A}$, except that, contrary to the latter, the parameter $\langle m\rangle_{e e}$ is not zero, and the mass-ratio $\frac{m_{2}}{m_{3}}$ is often larger than what we get in the $\mathbf{A}$ class.

\section{Singular models}

Patterns $\mathbf{S} 1, \mathbf{S} 2$ and S3: vanishing minors $\left(C_{31}, C_{11}\right),\left(C_{21}, C_{11}\right)$ and $\left(C_{31}, C_{21}\right)$ : The patterns $\mathbf{S} 1$ and S2 (which are related by the $T$ symmetry) lead to $m_{2}<m_{1}$, whence they are not acceptable. The pattern S3 lead to a degenerate non-acceptable model with $\left(m_{1}=m_{2}=m_{3}\right)$.

However, all these models can allow for one, and only one, model in which the neutrino mass matrix is singular. This happens only when $m_{3}=0$ and $\theta_{z}=0$. A vanishing $\theta_{z}$ is still consistent with experimental data as shown in eq. (15). In this pattern with vanishing $m_{3}$ we have $m_{1}=\sqrt{\Delta m_{\text {atm }}^{2}-\Delta m_{\text {sol }}^{2}}$ and $m_{2}=\sqrt{\Delta m_{\mathrm{atm}}^{2}}$. As to the parameters $\langle m\rangle_{e e}$ and $\langle m\rangle_{e}$, we have respectively

$$
\begin{aligned}
\langle m\rangle_{e e} & =\sqrt{m_{1}^{2} c_{x}^{4}+m_{2}^{2} s_{x}^{4}+2 m_{1} m_{2} c_{x}^{2} s_{x}^{2} c_{2 \rho-2 \sigma}}, \\
\langle m\rangle_{e} & =\sqrt{m_{1}^{2} c_{x}^{2}+m_{2}^{2} s_{x}^{2}} .
\end{aligned}
$$

The resulting mass matrix has the following elements, where the missing ones are related by symmetry $\left(M_{\nu}=M_{\nu}^{T}\right)$

$$
M_{\nu 11}=\left(m_{1} c_{x}^{2} e^{2 i \rho}+m_{2} s_{x}^{2} e^{2 i \sigma}\right)
$$




$$
\begin{aligned}
& M_{\nu 12}=s_{x} c_{x} c_{y} e^{-i \delta}\left(-m_{1} e^{2 i \rho}+m_{2} e^{2 i \sigma}\right), \\
& M_{\nu 13}=s_{x} c_{x} s_{y} e^{-i \delta}\left(m_{1} e^{2 i \rho}-m_{2} e^{2 i \sigma}\right), \\
& M_{\nu 22}=c_{y}^{2} e^{-2 i \delta}\left(m_{1} s_{x}^{2} e^{2 i \rho}+m_{2} c_{x}^{2} e^{2 i \sigma}\right), \\
& M_{\nu 23}=-c_{y} s_{y} e^{-2 i \delta}\left(m_{1} s_{x}^{2} e^{2 i \rho}+m_{2} c_{x}^{2} e^{2 i \sigma}\right), \\
& M_{\nu 33}=s_{y}^{2} e^{-2 i \delta}\left(m_{1} s_{x}^{2} e^{2 i \rho}+m_{2} c_{x}^{2} e^{2 i \sigma}\right) .
\end{aligned}
$$

The mixing angles $\theta_{x}$ and $\theta_{y}$ are constrained by data as given in eq. (15). However, there is no constraint on the phases $\delta, \rho$ and $\sigma$, thus for such a model there is no definite prediction for these phases. For the sake of illustration, we present a numerical value for the mass matrix for $\delta=75^{\circ}, \rho=30^{0}$ and $\sigma=30^{0}$, while $\theta_{x}$ and $\theta_{y}$ take their central value as given in eq. (15),

$$
M_{\nu}=m_{1}\left(\begin{array}{ccc}
0.497+0.862 i & -0.006+0.001 i & 0.005-0.001 i \\
-0.006+0.001 i & 0.106 \times 10^{-9}-0.546 i & -0.123 \times 10^{-9}+0.492 i \\
0.005-0.001 i & -0.123 \times 10^{-9}+0.492 i & 0.909 \times 10^{-10}-0.443 i
\end{array}\right) .
$$

\section{Failing cases}

Pattern F1, F2, F3, F4 and F5: The patterns F1, F2 and F3 are not acceptable since they lead to

$m_{2}<m_{1}$, where as the patterns $\mathbf{F} 4$ and $\mathbf{F} 5$ can not account for the neutrino oscillation since they give $m_{1}=m_{2}=m_{3}$. 


\begin{tabular}{|c|c|c|c|c|c|c|c|c|c|c|c|c|c|c|c|c|}
\hline Model & Minors & Hierarchy & $\theta_{x}$ & $\theta_{y}$ & $\theta_{z}$ & $\delta$ & $R_{\nu}$ & $\frac{m_{1}}{m_{3}}$ & $\frac{m_{2}}{m_{3}}$ & $\rho$ & $\sigma$ & $m_{3}$ & $\Delta m_{\mathrm{atm}}^{2}$ & $\Sigma$ & $\langle m\rangle_{e e}$ & $\langle m\rangle_{e}$ \\
\hline A1 & 33,32 & $\mathrm{~N}$ & 34 & 42 & 7 & 0 & 0.0298 & 0.060 & 0.180 & 0 & 90 & 0.052 & 0.0027 & 0.065 & 0 & 0.009 \\
\hline A2 & 22,32 & $\mathrm{~N}$ & // & 48 & // & 180 & // & // & // & // & // & // & // & // & // & // \\
\hline B3 & 33,31 & $\mathrm{~N}$ & 34 & 42 & 5 & 88.3 & 0.0325 & 0.807 & 0.814 & 179 & 178 & 0.085 & 0.0025 & 0.223 & 0.069 & 0.069 \\
\hline B4 & 22,21 & $\mathrm{~N}$ & // & 48 & // & 268.3 & // & // & // & // & // & // & // & // & // & // \\
\hline B4 & 22,12 & I & 34 & 42 & 5 & 90.4 & 0.0310 & 1.227 & 1.234 & 1.16 & 0.05 & 0.070 & 0.0026 & 0.242 & 0.086 & 0.086 \\
\hline B3 & 33,31 & I & // & 48 & // & 270.4 & // & // & // & // & // & // & // & // & // & // \\
\hline B5 & 33,12 & $\mathrm{~N}$ & 34 & 42 & 5 & 92 & 0.0337 & 0.802 & 0.809 & 1.4 & 2.34 & 0.082 & 0.0024 & 0.215 & 0.066 & 0.066 \\
\hline B6 & 22,13 & $\mathrm{~N}$ & // & 48 & // & 272 & // & // & // & // & // & // & // & // & // & // \\
\hline B6 & 22,13 & $\mathrm{I}$ & 34 & 42 & 5 & 89.83 & 0.033 & 1.234 & 1.241 & 179 & 0.26 & 0.066 & 0.0024 & 0.231 & 0.082 & 0.082 \\
\hline B5 & 33,12 & I & // & 48 & // & 269.83 & // & // & // & // & // & // & // & // & // & // \\
\hline D & 22,33 & $\mathrm{~N}$ & 34 & 41 & 5 & 52.7 & 0.033 & 0.512 & 0.534 & 14.35 & 128.95 & 0.058 & 0.0024 & 0.118 & 0.016 & 0.030 \\
\hline
\end{tabular}

V Table 3: The acceptable patterns for the two-vanishing minors. The minor corresponding to the index ( $i j)$ is the determinant of the sub-matrix obtained by deleting the $i^{\text {th }}$ line and the $j^{t h}$ column. All the angles (masses) are evaluated in degrees ( $\mathrm{eV}$ ) (the mark // means a value equal to what overheads it). 


\section{Symmetry realization}

All textures with zero minors discussed in this work can be realized in a simple way in models based on seesaw mechanism with a flavour Abelian symmetry. Zero minors in the non singular neutrino mass matrix $\left(M_{\nu}\right)$ are equivalent to zeros in the inverse mass matrix $\left(M_{\nu}^{-1}\right)$. This equivalence, however, is valid only for invertible mass matrices. In turn, eq. (1), tells us that, in case of diagonal neutrino Dirac mass matrices, zeros in $\left(M_{\nu}^{-1}\right)$ lead to zeros in $\left(M_{R}\right)$.

In order to construct the required leptonic mass matrices, we need three right-handed neutrinos $\nu_{R j}$, three right-handed charged leptons $l_{R j}$ and three left-handed lepton doublets $D_{L j}=\left(\nu_{L j}, l_{L j}\right)^{T}$, where $j$ is the family index running from 1 to 3 . As to the scalar sector, one Higgs doublet, the standard model SM Higgs, and many scalar singlets are required.

The underlying symmetry for building up mass matrices can be an Abelian discrete one as invoked in [15]. Following the same strategy, one can utilize $Z_{8}$ for constructing the model B3 which is characterized by vanishing minors $(33,31)$, and hence zeros at positions $(33,31)$ in $\left(M_{\nu}^{-1}\right)$. Under the action of $Z_{8}$, leptons of the first family remain invariant, those of the second family change sign, and those of the third get multiplied by $\omega=\exp \left(\frac{i \pi}{4}\right)$, whereas the SM Higgs doublet remains invariant. These assigned transformations under $Z_{8}$ automatically generate diagonal Dirac mass matrices for both charged leptons and neutrinos.

The bilinears $\nu_{R i} \nu_{R j}$ under $Z_{8}$, necessary for constructing the Majorana mass for right handed neutrino, transform as

$$
\left(\begin{array}{ccc}
1 & \omega^{4} & \omega \\
\omega^{4} & 1 & \omega^{5} \\
\omega & \omega^{5} & \omega^{2}
\end{array}\right),
$$

The $(1,1)$ and $(2,2)$ matrix elements of $M_{R}$ are $Z_{8}$ invariant, hence their corresponding mass terms are directly present in the Lagrangian. The $(1,2)$ matrix element requires the presence of a real scalar singlet (call it $\chi_{12}$ ) which changes sign under $Z_{8}$. The $(2,3)$ matrix element is generated by the Yukawa coupling to a complex scalar singlet (call it $\chi_{23}$ ) which gets multiplied by $\omega^{3}$ under $Z_{8}$. The other entries of $M_{R}$ remains zero in the absence of any further scalar singlets. The resulting right-handed Majorna mass matrix can be casted in the form,

$$
\left(\begin{array}{ccc}
\times & \times & 0 \\
\times & \times & \times \\
0 & \times & 0
\end{array}\right),
$$

where the cross sign denotes a non-vanishing element.

Out of fifteen possible models, nine of them A1, A2, B3, B4, B5, B6, S1, S2 and F3 can be constructed using $Z_{8}$ symmetry. The leftover models can be generated using $Z_{4}$ symmetry. Symmetry realizations for all models are summarized in Table. (4), where the transformation properties for each lepton family and needed scalar singlets are given for each model (for completeness, we stated even the phenomenologically non-successful models).

The question arises whether or not this seesaw enforcement of zero minors at high scale persists down to low scales. Actually, the renormalization group analysis shows that this is possible with one Higgs doublet [15], since the matrices $M_{\nu}$ at any two energy scales $\mu_{1}$ and $\mu_{2}$ are related by [20]

$$
M_{\nu}\left(\mu_{1}\right)=I M_{\nu}\left(\mu_{2}\right) I,
$$

where the matrix $I$ (which depends on $\mu_{1}$ and $\mu_{2}$ ) is diagonal, positive, and non-singular. It follows that any zero minor in $M_{\nu}$, at a given energy scale, remains zero at any other energy scale.

The models $\mathbf{S} 1, \mathbf{S} 2$ and $\mathbf{S} 3$ in the limit of vanishing $m_{3}$ and $\theta_{z}$ together give a singular mass matrix $M_{\nu}$. To produce such models of acceptable non-invertible textures within seesaw schemes, $M_{R}$ can not be singular otherwise the sea-saw mechanism would not work, and hence the only choice is to have a 


\begin{tabular}{|c||c||c|c|c|c|c|c|c|c|c|}
\hline \multicolumn{10}{|c|}{$Z_{8}$ Models } \\
\hline Model & Minors & $1_{F}$ & $2_{F}$ & $3_{F}$ & $\chi_{11}$ & $\chi_{12}$ & $\chi_{13}$ & $\chi_{22}$ & $\chi_{23}$ & $\chi_{33}$ \\
\hline B3 & 33,31 & 1 & -1 & $\omega$ & absent & -1 & absent & absent & $\omega^{3}$ & absent \\
\hline B5 & 33,12 & 1 & -1 & $\omega$ & absent & absent & $\omega^{7}$ & absent & $\omega^{3}$ & absent \\
\hline A1 & 33,32 & 1 & -1 & $\omega$ & absent & -1 & $\omega^{7}$ & absent & absent & absent \\
\hline F3 & 11,32 & $\omega$ & -1 & 1 & absent & $\omega^{3}$ & $\omega^{7}$ & absent & absent & absent \\
\hline A2 & 22,32 & 1 & $\omega$ & -1 & absent & $\omega^{7}$ & -1 & absent & absent & absent \\
\hline B4 & 22,12 & 1 & $\omega$ & -1 & absent & absent & -1 & absent & $\omega^{3}$ & absent \\
\hline S1 & 31,11 & $\omega$ & -1 & 1 & absent & $\omega^{3}$ & absent & absent & $\omega^{4}$ & absent \\
\hline B6 & 22,31 & 1 & $\omega$ & -1 & absent & $\omega^{7}$ & absent & absent & $\omega^{3}$ & absent \\
\hline S2 & 11,12 & $\omega$ & -1 & 1 & absent & absent & $\omega^{7}$ & absent & $\omega^{4}$ & absent \\
\hline \multicolumn{10}{||||}{} & \multicolumn{10}{|c||}{} & \multicolumn{1}{|c|}{$Z_{4}$ Models } & & & & \\
\hline F1 & 33,11 & $i$ & 1 & $-i$ & absent & $-i$ & absent & absent & $i$ & absent \\
\hline D & 22,33 & 1 & $i$ & $-i$ & absent & $-i$ & $i$ & absent & absent & absent \\
\hline F2 & 22,11 & $i$ & $-i$ & 1 & absent & absent & $-i$ & absent & $i$ & absent \\
\hline F4 & 31,32 & $i$ & $-i$ & 1 & -1 & absent & absent & -1 & absent & absent \\
\hline S3 & 31,12 & 1 & $i$ & $-i$ & absent & absent & absent & -1 & absent & -1 \\
\hline F5 & 32,12 & $i$ & 1 & $-i$ & -1 & absent & absent & absent & absent & -1 \\
\hline
\end{tabular}

Table 4: The symmetry realization for 15 patterns of two-vanishing minors. The index $1_{F}$ indicates the lepton first family and so on. The $\chi_{k j}$ denotes a scalar singlet which produce the entry $(k, j)$ of the right-handed Majorana mass matrix when acquiring a vev at the see-saw scale. The transformation properties, under the specified group, is listed below each lepton family and needed scalar singlets for each model. $\omega$ denotes $\exp \left(\frac{i \pi}{4}\right)$, while $i=\sqrt{-1}$.

singular Dirac neutrino mass matrix. A simple guess, which by no means excludes other possibilities, is where $M_{R}$ and $M_{D}$ have the following forms:

$$
M_{R}=\left(\begin{array}{ccc}
0 & \alpha & 0 \\
\alpha & \beta & 0 \\
0 & 0 & \gamma
\end{array}\right), \quad M_{D}=\left(\begin{array}{ccc}
D & 0 & A \\
0 & 0 & B \\
0 & 0 & C
\end{array}\right)
$$

where $A, B, C, D, \alpha, \beta$ and $\gamma$ are arbitrary parameters. The resulting neutrino mass matrix generated through seesaw mechanism takes the form,

$$
M_{\nu}=\left(\begin{array}{ccc}
-\frac{D^{2} \beta}{\alpha^{2}}+\frac{A^{2}}{\gamma} & \frac{A B}{\gamma} & \frac{A C}{\gamma} \\
\frac{A B}{\gamma} & \frac{B^{2}}{\gamma} & \frac{B C}{\gamma} \\
\frac{A C}{\gamma} & \frac{B C}{\gamma} & \frac{C^{2}}{\gamma}
\end{array}\right) .
$$

This matrix has a zero eigenvalue $\left(m_{3}=0\right)$ and vanishing minors $(31,11,12)$ as required. The symmetry realization for this construction could be done through a generic choice of the group $Z_{12} \times Z_{2}$ discussed in [21], which may not be the most economic way. The leptonic fields transform as

$$
\begin{array}{ccc}
l_{R 1} \rightarrow \theta l_{R 1}, & \nu_{R 1} \rightarrow \theta \nu_{R 1}, & \bar{D}_{L 1} \rightarrow \theta \bar{D}_{L 1}, \\
l_{R 2} \rightarrow \theta^{2} l_{R 2}, & \nu_{R 2} \rightarrow \theta^{2} \nu_{R 1}, & \bar{D}_{L 2} \rightarrow \theta^{3} \bar{D}_{L 2}, \\
l_{R 3} \rightarrow \theta^{5} l_{R 3}, & \nu_{R 3} \rightarrow \theta^{5} \nu_{R 3}, & \bar{D}_{L 3} \rightarrow \theta^{8} \bar{D}_{L 3},
\end{array}
$$

where $\theta=e^{\frac{i \pi}{6}}$. Hence, the bilinears $\bar{D}_{L j} l_{R k}, \bar{D}_{L j} \nu_{R k}$ and $\nu_{R j} \nu_{R k}$ transform as

$$
\bar{D}_{L j} l_{R k} \sim \bar{D}_{L j} \nu_{R k} \sim\left(\begin{array}{ccc}
\theta^{2} & \theta^{3} & \theta^{6} \\
\theta^{4} & \theta^{5} & \theta^{8} \\
\theta^{9} & \theta^{10} & \theta
\end{array}\right), \quad \nu_{R j} \nu_{R k} \sim\left(\begin{array}{ccc}
\theta^{2} & \theta^{3} & \theta^{6} \\
\theta^{3} & \theta^{4} & \theta^{7} \\
\theta^{6} & \theta^{7} & \theta^{10}
\end{array}\right) \text {. }
$$


To achieve a diagonal charged lepton mass matrix, only three scalar Higgs doublets are needed which are denoted by $\Phi_{11}, \Phi_{22}$ and $\Phi_{33}$. Under the action of $Z_{12}$ these scalar doublets get respectively multiplied by $\theta^{10}, \theta^{7}$ and $\theta^{11}$. The Dirac and Majorana neutrino mass matrices in eq. (57) can be achieved equally by introducing scalar Higgs fields with suitable transformation properties, namely four scalar doublets $\tilde{\Phi}_{11}, \tilde{\Phi}_{13}, \tilde{\Phi}_{23}$, and $\tilde{\Phi}_{33}$, being multiplied respectively by $\theta^{10}, \theta^{6}, \theta^{4}$ and $\theta^{11}$ under $Z_{12}$, for the Dirac mass term, and three scalar singlets $\chi_{12}, \chi_{22}$ and $\chi_{33}$, multiplied respectively by $\theta^{9}, \theta^{8}$ and $\theta^{2}$ under

$Z_{12}$, for the Majorana mass term. The $\tilde{\Phi}_{j k}$ scalar Higgs doublet and $\nu_{R j}$ change sign under $Z_{2}$, while all other multiplets remain invariant. It is important to notice that the scalar Higgs doublets take vacuum expectation values (vev) at the electro-weak scale, while scalar singlets acquire vevs at the seesaw scale.

\section{Summary and conclusions}

We studied all the possible patterns of Majorana neutrino mass matrices with two independent vanishing minors.

For the possible fifteen cases, we found seven patterns (A1, A2, B3, B4, B5, B6 and D) able to accommodate current data, without need to tune the input parameters.

Five patterns (S1, S2, F1, F2 and F3) lead to $m_{2}$ smaller than $m_{1}$ and thus are non-acceptable. Three cases (S3, F4, and F5) predict degeneracy of masses $m_{1}=m_{2}=m_{3}$, and thus can not make room for the oscillation phenomena.

The acceptable patterns can be classified into three categories (A, $\mathbf{B}$ and $\mathbf{D})$. The classes $\mathbf{A}$ and $\mathbf{D}$ allow only for N-type hierarchy, whereas the class $\mathbf{B}$ has room for both N- and I-type of hierarchies.

The six cases (A1, A2, B3 and B4) appear as 'two-zero' textures, and the corresponding analytical expressions agree with [9]. The other three two-zero textures allowed by current data, and denoted by (B1, B2 and $\mathbf{C})$ in [9], are not reproduced as two-vanishing minors textures. On the other hand, there are three two-vanishing minor textures $(\mathbf{B 5}, \mathbf{B} 6$ and $\mathbf{D})$ which are allowed by data and do not show up as two-zero textures. This classification of patterns coincides exactly with the results of [15]. However, as mentioned in the introduction, the analytical parts of the two approaches are not completely identical, so that one needs not to assume an invertible neutrino mass matrix in the 'vanishing minor' approach.

In all the successful models, the non oscillation parameters $\langle m\rangle_{e},\langle m\rangle_{e e}$ and $\Sigma$ are consistent with the bounds given in eq. (15). In addition, $\langle m\rangle_{e},\langle m\rangle_{e e}$ and $m_{3}$ have the same order of magnitude. The mass sum parameter is always constrained to be $\Sigma \leq 0.242 \mathrm{eV}$ which is safe with the cosmological bound in eq. (15).

There are some characteristic features which might differentiate the classes from each other. First, for the class A patterns, there is an acute hierarchy in the mass spectrum since the combination $s_{z} t_{y}$ appears in the mass ratio masses making the hierarchy sharp for small enough $s_{z}$. The hierarchy in class $\mathbf{B}$ is less sharp and the three masses are all of the same order of magnitude, since $t_{y}$ alone appears in the leading term of the expansion of the mass ratios. Second, in the class $\mathbf{A}$ there is a lower bound on $\theta_{z}$, whereas no such bound in classes $\mathbf{B}$ and $\mathbf{D}$. Third, since the Dirac angle $\delta$ is not constrained in the class $A$ or $D$, whereas it is likely to be around right angles in the class $\mathbf{B}$, then it is in this latter class that $\mathrm{CP}$ is likely to be maximally violated. Fourth, there is no signal for neutrinoless double beta decay in the class $\mathbf{A}$, while the corresponding parameter $\langle m\rangle_{e e}$ does not vanish in the classes $\mathbf{B}$ and $\mathbf{D}$.

Table (2) presents the key signatures involving $\left(\delta, \theta_{y}\right)$ allowing to distinguish among the patterns of the class $\mathbf{B}$. Also, there is a 'correlation' between these two parameters in class $\mathbf{D}$ in that $\theta_{y}$ being in first (second) octant leads to $c_{\delta}>0\left(c_{\delta}<0\right)$, whereas no such relation in class $\mathbf{A}$.

New results are obtained in case of non-invertible mass matrix which can not be recovered in [15]. Those correspond to the patterns $\mathbf{S 1}, \mathbf{S} 2$ and $\mathbf{S 3}$. For these models, we only find a consistent solution when both $m_{3}$ and $\theta_{z}$ vanish, a solution that accommodates $\theta_{x}$ and $\theta_{y}$ to be in their acceptable range given in eq. (15), while the phases $\delta, \sigma$ and $\rho$ are not constrained. Peculiarly enough, the three cases 
have identical mass matrix as given in eq. (53), and thus it would be difficult to distinguish between the three models in this limit of vanishing $m_{3}$ and $\theta_{z}$. An interesting thing about these three models is that they correspond to two-zero textures before taking the limit of vanishing $m_{3}$ and $\theta_{z}$, whereas they cease to be so after taking the limit where they become only characterized by vanishing two minors and the whole determinant.

Concluding remarks are in order. First, for all patterns the leading terms in the expansion of the angles $(\rho, \sigma)$ in powers of $\left(\sin \left(\theta_{z}\right)\right)$ are either $\delta$ or $\frac{\delta}{2}\left(\bmod \frac{\pi}{2}\right)$. Second, the models of the classes $\mathbf{B}$ and $\mathbf{D}$, do not have room for the atmospheric mixing angle to be maximal $\left(\frac{\pi}{4}\right)$, otherwise the parameter $R_{\nu}$ would become too large. Third, all models can be generated in the frame work of flavour Abelian discrete symmetry, and additional scalar fields with appropriate transformation properties, implemented in seesaw schemes.

\section{Acknowledgements}

Both authors would like to thank L. Lavoura for drawing our attention to his work. One of the authors, E. I. Lashin would like to thank both of A. Smirnov and S. Petcov for useful discussions. Part of this work was done within the associate scheme of ICTP.

\section{References}

[1] Y. Fukuda et al., Phys. Lett. B 436, 33 (1998); Phys. Rev. Lett. 81, 1562 (1998). For a review, see: C.K. Jung, C. McGrew, T. Kajita, and T. Mann, Ann. Rev. Nucl. Part. Sci. 51, 451 (2001).

[2] S. M. Bilenky, and B. Pontecorvo, Phys. Rep 41, 225 (1978);

S. M. Bilenky and S. T. Petcov, Rev. Mod. Phys. 59, 671, (1987)

[3] Particle Data Group, K. Hagiwara et al., Phys. Rev. D 66, 010001 (2002).

[4] SNO Collaboration, Q.R. Ahmad et al., Phys. Rev. Lett. 89, 011301 (2002); Phys. Rev. Lett. 89, $011302(2002)$.

[5] KamLAND Collaboration, K. Eguchi et al., Phys. Rev. Lett. 90, 021802 (2003).

[6] K2K Collaboration, M.H. Ahn et al., Phys. Rev. Lett. 90, 041801 (2003).

[7] CHOOZ Collaboration, M. Apollonio et al., Phys. Lett. B 420, 397 (1998); Palo Verde Collaboration, F. Boehm et al., Phys. Rev. Lett. 84, 3764 (2000).

[8] P.H. Frampton, S.L. Glashow, and D. Marfatia, Phys. Lett. B 536, 79 (2002).

[9] Z.Z. Xing; Phys. Lett. B 530 (2002), 159-166

[10] S. Dev, S. Kumar, S. Verma and S. Gupta; Phys. Rev. D 76, 013002 (2007)

[11] A. Merle and W. Rodejohann; Phys. Rev. D 73, 073012 (2006)

[12] Z.Z. Xing; Phys. Rev. D 69, 013006 (2004)

[13] E. Ma; Phys. Rev. D 71, 111301 (2005)

[14] S.-L. Chen, M. Frigero and E. Ma; Nucl. Phys. B 724, 423 (2005)

[15] L. Lavoura; Phys. Lett. B 609, 317 (2005) 
[16] H. A. Alhendi, E. I. Lashin and A. A. Mudlej, Phys. Rev. D 77, 013009 (2008);hep-ph/0708.2007

[17] G. L. Fogli et al., Prog. Part. Nucl. Phys. 57, 742 (2006)

[18] H. V. Klapdor-Kleingrothaus et al., Mod. Phys. Lett. A 16 (2001) p. 2409

[19] J. Jenkins, hep-ph/0808.1702

[20] P.H. Chankowski, Z. Płuciennik, Phys. Lett. B316, 1993, 312

[21] W. Grimus, A.S. Joshipura, L. Lavoura and M. Tanimoto, Eur. Phys. J. C 36, 227 (2004) 